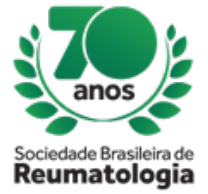

\title{
TREATMENT OF LUPIC NEPHRITIS: RESULT OF 5 YEARS EXPERIENCE IN A SCHOOL HOSPITAL
}

Lorena Bossoni Miosso Marchiotti (Universidade Estadual de Maringá, Maringá, PR, Brasil), Fernanda

Maria Borghi (Universidade Estadual de Maringá, Maringá, PR, Brasil), Sueny de Paula Monarin

(Universidade Estadual de Maringá, Maringá, PR, Brasil), Pedro Henrique Pelissari (Universidade

Estadual de Maringá, Maringá, PR, Brasil), Vinícius Luis de Freitas Souza (Universidade Estadual de maringá, Mafingá, PR, Brasil), Vivian Marques Herrera (Universidade Estadual de Maringá, Maringá, PR Brasil), Marcos Madeira de Lima (Universidade Estadual de maringá, Maringá, PR, Brasil), Jessica Silva Gongora (Universidade Estadual de maringá, Maringá, PR, Brasil), Paulo Roberto Donadio (Universidade Estadual de maringá, Maringá, PR, Brasil)

\section{BACKGROUND}

Systemic lupus erythematosus (SLE) is a chronic autoimmune inflammatory disease whose etiopathogenesis involves multiple genes, hormonal and environmental factors. One of the most serious complications of SLE is lupus nephritis, which is a poor prognosis.

\section{MATERIALS AND METHODS}

Retrospective analyzis of the results of the treatment performed in patients with lupus nephritis between January 2012 and December 2016, attended at a University Hospital.

\section{RESULTS}

202 patients with SLE, 59 (29.20\%), with diagnosis of nephritis. 30 diagnosed and treated during the proposed 5-year period. 29 submitted to renal biopsy, except 1 for being pregnant. Female (100\%); mean age 35.7, minimum 22 years and maximum 52 years; 4 smokers (13.3\%); 9 hypertensive (30\%), 3 diabetic (10\%), 2 hypothyroid (6.66\%), 2 dyslipidemic (6.66\%), and 14 without comorbidities (46.66\%). Pathologic anatomy, according to the International Society of Nephrology / Renal Pathology Society 2003 Lupus Nephritis Classification: Normal: 2 patients (6.89\%); Class I: Minimal mesangial lupus nephritis: 3 patients (10.34\%); Class II: proliferative mesangial lupic nephritis: none; Class III: Focal lupus nephritis: 2 patients (6.89\%); Class IV: Diffuse lupus nephritis: 16 patients (55.17\%); Class V: membranous lupus nephritis: 6 patients (20.68\%); Class VI: Advanced sclerosis: none. All in use of hydroxychloroquine and antiproteinuric medications. Treatment performed: pulse therapy with methylprednisolone for 3 days, followed by monthly pulse therapy with cyclophosphamide for 6 months, in all patients in class III, IV and V; maintenance with azathioprine or mycophenolate mofetil. The others were treated with corticosteroids and azathioprine. None received induction with mycophenolate mofetil. $16(53.33 \%)$ achieved complete remission (CR); 8 (26.66\%), partial remission; 4 (13.33\%) refractory to the treatment, needing to change medication (tacrolimus or cyclosporine); and, 2 (6.66\%) did not complete induction with cyclophosphamide due to repeat infections.

\section{CONCLUSION}

The main objective of the treatment proposed by the Consensus of the Brazilian Society of Rheumatology for the diagnosis, management and treatment of lupus nephritis is to achieve CR, which is associated with good prognosis in the long term. However, less than $50 \%$ of patients with lupus nephritis get CR after six months of treatment. The experience presented here reached $53 \% \mathrm{CR}$ after 1 year of treatment. The data presented demonstrated that the biopsy and classification of the histological lesion is fundamental for the therapeutic conduction according to the current protocols, and obtaining good results, in agreement with other specialized centers. 